\title{
AN OVERVIEW OF \\ THE MICHIGAN POSITRON MICROSCOPE PROGRAM
}

\author{
D. W. Gidley, W. E. Frieze, T. L. Dull, \\ G. B. DeMaggio, E. Y. Yu, H. C. Griffin, \\ M. Skalsey, R. S. Vallery, and B. D. Wissman \\ Department of Physics, ${ }^{*}$ Department of Chemistry \\ University of Michigan \\ Ann Arbor, Michigan 48109
}

\begin{abstract}
An overview of the Michigan Positron Microscope Program is presented with particular emphasis on the second generation microscope that is presently near completion. The design and intended applications of this microscope will be summarized.
\end{abstract}

\section{INTRODUCTION}

The invention of a variety of positron microscopes was reported in 1988 . A transmission positron microscope (TPM), similar in operation to its electron counterpart, was demonstrated at the University of Michigan. A scanning positron microprobe (SPM) was operated at Brandeis. Two different versions of a positron reemission microscope (PRM) were invented later in 1988 at Michigan ${ }^{3}$ and at Brandeis. The PRM, SPM, and to a lesser extent the TPM, display totally unique image contrast mechanisms and thereby provide the materials researcher with a new view of phenomena that are not easily visible with existing microscopies. Positrons exhibit such behavior as trapping in open-volume defects as small as monovacancies, reemission from a surface due to a negative work function, electron capture to form positronium (similar to hydrogen formation), and annihilation with electrons yielding readily detectable gamma rays that reveal the annihilating pair's momentum. All of these unique positron properties lead to new forms of contrast in positron micrographs.

For the last three years the University of Michigan positron group has been funded by DoE's Advanced Energy Projects to design, construct, and begin testing a second generation microscope with greatly expanded capabilities as a PRM, SPM, and as an electron microscope. In the next section we will present a more detailed progress report on this new microscope-its design, modes of operation, and contrast mechanisms. At this point we will only present an overview of our microscope program which consists of four highly interrelated projects:

1. The Microscope. Construction of the second generation UHV microscope is the central element in the program. Designed to take advantage of thick, robust target samples, it is built as a reflection-style PRM compared to the Brandeis transmission-style $\mathrm{PRM}^{4}$ which is based on thin film targets. As an SPM it is designed to operate with many existing positron techniques to generate the scanned signal. In addition, electron techniques of secondary electron microscopy, scanning Auger spectroscopy, and even low energy electron microscopy (LEEM) have been incorporated. 
2. ${ }^{58} \mathrm{Co}$ Sources. To provide positron sources for the microscope we have been working on a totally in-house process for fabrication of thin ${ }^{58} \mathrm{Co}$ radioactive sources. Ni targets are irradiated in the University's Ford Nuclear Reactor. Co is chemically separated and then electroplated on a $3 \mathrm{~mm}$ diameter spot prior to encapsulation. In August a 40 gram Ni plate was completely processed and tested in a positron beam as a mock test of what would have been a $1 \mathrm{Ci}^{58} \mathrm{Co}$ source if the $\mathrm{Ni}$ had been left in the reactor for several months. It was successful and we are confident that we will have such sources by summer 1993. By 1994 we hope to extend the process by roughly an order of magnitude.

3. INEL Collaboration. Realizing the longer-term need for even higher intensity sources we are actively collaborating with the Idaho National Engineering Laboratory (INEL) to scale up this ${ }^{58} \mathrm{Co}$ program using their EBR II reactor to the level of 10,000 to $100,000 \mathrm{Ci}$. The intense fast neutron flux of EBR II accounts for over a factor of 100 in the scaling. The goal of this collaboration is to construct (within 6 years) a third generation microscope facility capable of real-time imaging with ultimate positron microscope resolution. The design and intended applications of this facility are to be determined by our experience with the present microscope.

4. Applications. We have active non-microscopic surface physics and materials research programs utilizing positrons that are generating applications for the microscope effort. We have used positron beams to explore thin films with reemitted positron spectroscopy. In particular, we have carried out studies of pseudomorphic growth, interdiffusion alloying, ${ }^{6}$ near surface defects, ${ }^{7}$ positron tunneling, ${ }^{5,8}$ positron mean free paths, ${ }^{6}$ and silicide thermal reaction dynamics. ${ }^{9}$ All of these processes affect contrast in a PRM. We are also studying fatigue initiation in glassy polymers using positron lifetime techniques ${ }^{10}$ and we are in the process of extending our slow positron beam techniques to include lifetime and Doppler broadening analysis.

With basic construction of the microscope completed and with $1 \mathrm{Ci}^{58} \mathrm{Co}$ sources expected to be ready this summer, we will begin exploring a broad-based program in materials research and engineering, condensed matter physics, and biological science. The eventual need for more intense beams can be satisfied in the short term by improving our in-house source technique to $10 \mathrm{Ci}$ and in the long run through our collaboration with INEL. In the meantime it is essential to explore those applications where positron microscopy can uniquely yield new information. It is precisely this goal that the present microscope is aimed at, with particular emphasis on materials research.

\section{DESIGN AND FEATURES OF THE MICROSCOPE}

A schematic representation of our second generation microscope is shown in Figure 1. The microscope incorporates both positrons and electrons because it can initially be difficult to interpret positron microscope images taken alone. Thus it is very important to be able to obtain well-characterized electron images of a sample in situ. In both cases, there are two possible imaging modesmagnifying mode and scanning mode. We summarize the charactistics of these various operational configurations below, and Table 1 summarizes some of the primary advantages and features the new microscope will have. 


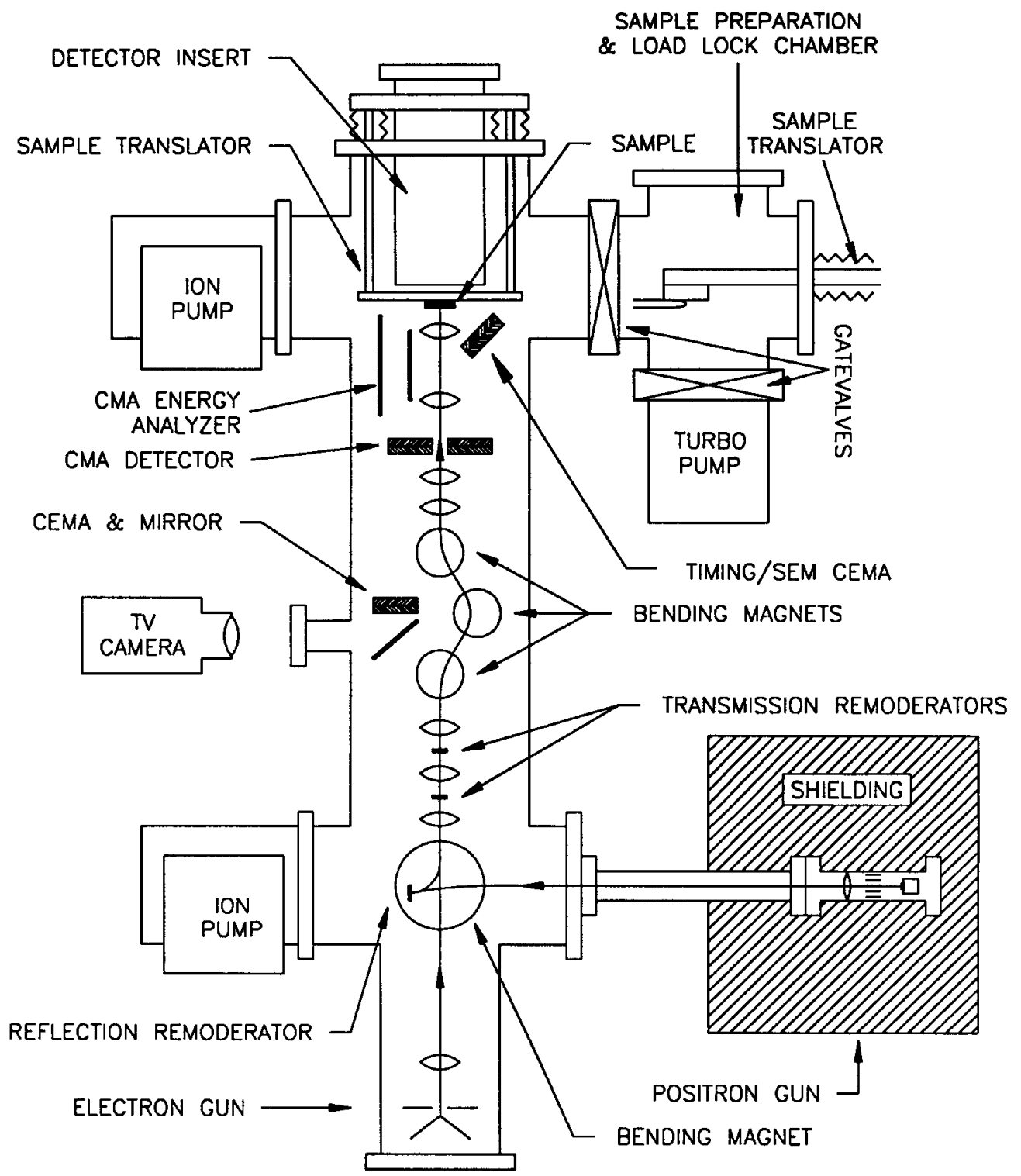

Fig. 1. Schematic Representation of the Microscope This figure illustrates the basic design features of our positron microscope (not to scale). It shows the positron and electron guns, the three remoderators, the optics and image detector necessary for magnification mode, and some of the other detectors necessary for the various signals of scanning mode. 


\section{Table 1: Advantages and Features of the Microscope}

Summary of some of the features our new positron microscope will have. In some cases, different features depend on the operating mode chosen. (For example, depth profiling and surface sensitivity appear contradictory, but in fact just depend on the choice of scanning mode vs. magnifying mode.)

- Multiprobe Capability Multiple modes of microscopy will be possible: magnifying and scanning positron microscopy, LEEM, SEM, and SAM.

- Defect Sensitivity

Positrons offer the most sensitive measure of defect concentrations available; they are directly sensitive to the presence of lattice disorder.

- Defect Specificity

Microscope signals can differentiate among various defect types, yielding simultaneous information on several concentrations.

- Depth Profiling

Different implantation depths for different incident beam energies permit studies at specified depths.

- Surface Sensitivity

Some operating modes (such as positron tunneling microscopy) are highly surface specific, permitting study of purely surface phenomena.

- High Resolution

Ultimate lateral resolution in magnifying mode should be near $10 \AA$. Submonolayer depth resolution is possible in tunneling mode.

- Thick Samples

Samples can be used directly with no thinning; standard thick-sample preparation techniques and electron spectroscopies can be employed.

- In Situ Capability

- Non-Destructivity

Positron microscopy may be used simultaneously with sample treatments such as heating, evaporative coating, or application of electrical signals.

The small rates of positron beams require instrumentation sensitive to currents in the $10^{-15} \mathrm{~A}$ range or below, thus reducing sample damage to a minimum.

- High Vacuum

Base pressure in the microscope should be in the $10^{-9}$ torr range. 
1. Positron Magnifying Mode. In this mode, positrons implanted in a sample are reemitted because of the negative work function. The reemitted positrons are then accelerated, magnified, and focused on an image detector. Any physical process which affects the probability of reemission, the energy, or the angular distribution can produce contrast in such an image. Magnifying mode should permit ultimate lateral resolutions near $10 \AA$, and can be performed in a depth profiled version (by varying the implantation energy of the incident positrons) up to a maximum depth of several thousand $\AA$. This mode is often referred to as a positron reemission microscope, PRM.

2. Positron Scanning Mode. This method, analogous to scanning electron microscopy, involves a small diameter beam rastered across the sample while a signal of some sort is collected in synchronization. Positrons permit a wide variety of signals, including Doppler shift in the energy of the annihilation gamma rays, lifetime of the positrons in the sample prior to annihilation, and number of emitted positronium atoms. Lateral resolutions in scanning positron microscopy are limited by diffusion-induced spreading of the beam spot to roughly $1000 \AA$, but depth profiling is possible up to a maximum depth of several $\mu \mathrm{m}$.

3. Electron Microscopies. Both magnifying and scanning mode will also be possible using electrons. Scanning electron microscopy and scanning Auger microscopy will be our primary traditional techniques for sample characterization. Fortunately, the optical system required for magnifying mode positron microscopy is identical to that required to perform the new technique of low energy electron microscopy (LEEM). ${ }^{11}$ In this technique, an image is formed using the electrons from a single diffraction spot at an incident energy typically well below $100 \mathrm{eV}$. In effect, LEEM is imaged LEED with lateral resolution better than $100 \AA$. It has generated considerable excitement in the surface physics community as a surface probe with sub-monolayer depth resolution combined with the capability of producing real-time images of dynamic processes. Our instrument will be able to perform LEEM with only minimal changes in the voltage supplies of the lenses.

\section{APPLICATIONS IN SURFACE STUDIES}

There are many possible applications of positron microscopy that we would like to explore over the next several years. Some of the most promising cases are mentioned below. They are ordered according to the operational mode of the microscope: either positron reemission microscopy (magnifying mode) or defect imaging (scanning mode).

In reemission mode we expect the microscope to have unique contrast for thin films and overlayers, as well as sensitivity to surface structure, impurity coverage, and near-surface defect distribution. One very interesting case occurs when an overlayer has a higher positron energy level than the substrate, thus forming a barrier for emission from below. If the overlayer is thin enough, emission can occur by tunneling. Positron tunneling microscopy ${ }^{8}$ should thus permit studies of overlayer growth and structure in suitable systems with submonolayer depth resolution. Some particular examples of studies we would like to initiate using magnifying mode are:

1. Growth Mechanisms and Pseudomorphism. Many of the phenomena associated with heteroepitaxial growth should be visible using positrons. Islanding, pin-holes, thickness variations and the like should be readily visible in 


\section{Michigan Positron Microscope Program}

reemitted positron images. Even in cases where there may be no topographical contrast, physical processes that change the positron energy level of the overlayer such as pseudomorphic growth and interdiffusion alloying can produce contrast. For example in the $\mathrm{Ni}$ on $\mathrm{Cu}$ system, we have observed ${ }^{5}$ that strained growth can eliminate the $0.5 \mathrm{eV}$ energy barrier to positron emission normally presented by a $\mathrm{Ni}$ overlayer. We would like to acquire micrographs at various $\mathrm{Ni}$ film thicknesses to study the commensurent-to-incommensurent transition that occurs near the critical thickness (bright areas would be under strain, dark areas would be relaxed with strain localized at the misfit dislocations).

2. Surface Catalysis. Positron microscopy may be very useful in studying model systems in chemical surface catalysis where traditional electron microscopies are known to be inadequate, namely low $\mathrm{Z}$ adsorbates on high $\mathrm{Z}$ substrates. Carbon and sulfur for example, do not have sufficiently high electron scattering cross sections to be observed on highly scattering, high $\mathrm{Z}$ substrates like $\mathrm{Ru}$ and $\mathrm{Pt}$. However, the positron emission process is independent of the thickness of the substrate, depending only on conditions near the surface. We should be able to observe the presence of adsorbed species by such mechanisms as trapping or scattering of the reemitted positrons, or changes in the positronium formation probability. LEEM may also provide complementary new information on these systems.

3. Thin Film and Interface Reaction Dynamics. The material specificity of positron reemission should be useful in studying the growth dynamics of systems such as metal silicide films produced by thermal reaction of metal films grown on silicon substrates. We have shown that all three major stoichiometries of the $\mathrm{Co}$ and $\mathrm{Ni}$ silicides have different positron work functions, and hence can be clearly distinguished in reemitted positron spectra. With depth profiling we should be able to observe the $3 \mathrm{D}$ growth profile of the reaction.

\section{APPLICATIONS IN DEFECT MAPPING}

As a scanning defect microprobe the microscope will find application in a broad range of defect nucleation/damage initiation studies. Because positrons directly detect disorder in a crystal (as opposed to traditional techniques such as diffraction measurements which look for small departures from perfect order), they are the most sensitive probes of defects available. They are able to detect monovacancies, for example, at concentrations as small as 1 in $10^{7}$. The wealth of signals available in scanning positron microscopy should permit detailed maps of the defects in a sample, maps which can be extended to three dimensions by depth profiling. Some particular examples of studies we would like to initiate using scanning mode are:

1. Electromigration Induced Voids. The initiation stages in the growth of electromigration induced voids in the conducting gateways of microelectronic devices are invisible with present electron techniques. High energy SEM can resolve such voids at the $0.1-1 \mu \mathrm{m}$ level, but there is no hope of observing the nucleation phase at the $10 \AA$ level. Defects of such a size are efficient positron traps, and hence will be visible in a positron micrograph. Thus we should be able to detect the defect nucleation phase of these voids well before they threaten the operation of the device, and at a size that is small even compared to the next several generations of downsized microelectronic devices. 
2. Corrosion Initiation. A phenomenon similar to the growth of electromigration induced voids in device interconnects is the pitting of passivating oxide layers during corrosion of metals. In this case, impurity ions pair with vacancies to form Frenkel type defects. These provide the charged species that migrate under the influence of internal fields to agglomerate as voids that eventually become corrosion pits. Again, positrons should be able to map defects in the early initiation phase when current microscopies see nothing and before complete failure of the passivation. Here the ability to image thick samples is a distinct advantage. LEEM may also offer exciting new possibilities in this field.

3. Fatigue Initiation in Polymers, Ceramics, and Composites. For several years, we have been participating in a program (funded by the Division of Materials Science of DoE) of non-microscopic studies of polymer fatigue, using (among other techniques) positron lifetimes. Our measurements ${ }^{10}$ have indicated that the physical changes induced by fatigue in glassy polymers such as polycarbonate are probably localized within a few $\mu \mathrm{m}$ of a very few nucleation sites. These sites eventually become visible as crazes and cracks, but at this point the sample is within $20 \%$ of the end of its useable life. The initiation phase, which is nominally $80 \%$ of the sample's fatigue lifetime, is invisible to conventional microscopies. Again, positron lifetime microscopy provides us with a technique for observing the nucleation of smaller defects as they evolve or coalesce into larger voids that become cracks. No other technique is sensitive to such small voids. A corollary study is planned for crach propagation in polymers, whereby the positron microprobe would be scanned in front of the crack to search for void coalescence and its spatial correspondence with the visible crack. This may provide the first firm evidence for microstructural changes at the $10 \AA$ level induced by the propagating crack.

We suspect the positron microscope will find similar uses in some composite, ceramic, and ceramic-fiber-composite materials where crack formation is the dominant fatigue failure mechanism. These high performance materials in terms of strength, rigidity, or durability are of particular interest in the transportation and utility industries.

4. Radiation Damage. Crack formation is not limited to polymers of course, nor is the instigating agent limited to fatigue. Another interesting application is to radiation damage in metals. The abilities of a scanning positron microscope to map defects, to differentiate among different types of defects, and to nondestructively examine samples as the damage evolves, should be invaluable in the study of the complex defect dynamics that precede failure in materials damaged by radiation.

\section{CONCLUSION}

We anticipate completion of the basic microscope early in 1993. Additional capabilities will continue to be added to the instrument thereafter, but the ability to acquire images should begin at that time. The thrust of our research over the next several years is to explore the widest range of applications possible and to develop the most promising of them. Our main construction projects will be a sample manipulator/load lock system and a separate sample preparation chamber, with outfitting dictated by the needs of the particular applications under study. In the next several years we should have a much clearer assesment of the capability of positron microscopy to provide unique information in material systems of scientific and technological importance. 


\section{Michigan Positron Microscope Program}

The construction of the second generation microscope was funded by DoE Grant DE-FG02-90ER12103 and by the Office of the Vice President for Research of the University of Michigan. Most of the ${ }^{58} \mathrm{Co}$ source development project has been funded by NSF Grant PHY-9119899, with some support from INEL. The application work has been mainly funded by NSF Grant DMR-9003987, with polymer studies funded by DoE Grant DE-FG02-88ER45366. This support is gratefully acknowledged.

\section{REFERENCES}

1. James Van House and Arthur Rich, Phys. Rev. Lett. 60, 169(1988).

2. G. R. Brandes, K. F. Canter, T. N. Horsky, and P. H. Lippel, Rev. Sci. Instrum. $59,228(1988)$.

3. James Van House and Arthur Rich, Phys. Rev. Lett. 61, 488(1988).

4. G. R. Brandes, K. F. Canter, and A. P. Mills, Jr., Phys. Rev. Lett. 61 , 492(1988).

5. D. W. Gidley, Phys. Rev. Lett. $62,811(1989)$.

6. D. W. Gidley, W. E. Frieze, Phys. Rev. Lett. 60 , 1193(1988).

7. D. W. Gidley, A. R. Köymen, T. W. Capehart, Phys. Rev. B, 37, 2465(1988).

8. W. E. Frieze, D. W. Gidley, B. D. Wissman, Solid State Comm., 74, 1079(1990).

9. B. D. Wissman, W. E. Frieze, D. W. Gidley, to be published Phys. Rev. $\mathrm{B}$, see paper this proceedings.

10. L. Liu, A. Yee, D. W. Gidley, Journal of Polymer Science: Part B, Polymer Physics $30,231(1991)$.

11. W. Telieps and E. Bauer, Ultramicroscopy, 17, 57(1985) and W. Telieps, Appl. Phys. A, 44, 55(1987). 